\title{
Völkerrechtlicher Naturschutz und nachhaltige Entwicklung in Übersee
}

\author{
Von UlfMarzik
}

\section{Einleitung}

"Entwicklung kann nicht sichergestellt werden ohne Bewahrung - Bewahrung nicht ohne Entwicklung." Diese auf den ersten Blick nicht aufsehenerregende These enthielt bereits die World Conservation Strategy des Umweltprogramms der Vereinten Nationen (UNEP) im Jahr 1980, ${ }^{1}$ die von den Umweltorganisationen IUCN und WWF vorbereitet worden war ${ }^{2}$ und die am Anfang der Diskussion um das Konzept der nachhaltigen Entwicklung steht. ${ }^{3}$

5 Jahre nach der Weltkonferenz über Umwelt und Entwicklung in Rio de Janeiro, 25 Jahre nach der Konferenz der Vereinten Nationen über die Umwelt des Menschen in Stockholm und nach fast 30 Jahren VERFASSUNG UND RECHT IN ÜBERSEE (VRÜ) gilt es, eine Bemerkung in Erinnerung zu rufen, die selbstverständlicher nicht ausfallen konnte, die in ihrer Umsetzung aber eines der brennendsten globalen Probleme markiert: Den Grundkonflikt zwischen dem Ziel der Entwicklung und dem richtigen - das soll heißen: verträglichsten Weg dorthin, den wir innerstaatlich, scheinbar harmlos, als das Spannungsverhältnis von Nutzung und Schutz der natürlichen Lebensgrundlagen ${ }^{4}$ umschreiben. Einige Vorbemerkungen sollen die Orientierung erleichtern.

1 International Union for the Conservation of Nature and Natural Resources, United Nations Environment Programme, World Wildlife Fund, World Conservation Strategy, Gland 1980; Martin W. Holdgate et al., Umwelt - weltweit. Bericht des Umweltprogramms der Vereinten Nationen (UNEP) 1972 - 1982, Berlin, 1981, S. 1.

Zur Entstehung vgl. Cyrille de Klemm, Clare Shine, Biological Diversity Conservation and the Law, IUCN, Gland 1993, S. 3.

Vgl. Kamal Hossain, Evolving principles of sustainable development and good governance, in: Konrad Ginther, Erik Denters, Paul J. I. M. de Waart (eds.), Sustainable Development and Good Governance, Dordrecht 1995, S. 17; insbesondere auch, zusammen mit den übrigen Beiträgen des Bandes, zu den gesellschaftlichen, wirtschaftlichen, politischen und staatsrechtlichen Aspekten des Konzeptes.

4

Zum innerstaatlichen Schutz der natürlichen Lebensgrundlagen vgl. die Formulierung in Art. 20a GG. 
Das Umweltrecht ist längst als Querschnittsrecht erkannt. Es betrifft wesentliche Aspekte nahezu aller Lebensbereiche, ohne einem Lebensbereich, nicht einmal dem des Menschen, ${ }^{5}$ eindeutig zuzuordnen zu sein. Die Befassung mit ihm verlangt von dem Betrachter nicht nur die Erkenntnis der Komplexität der - rechtlichen und tatsächlichen ${ }^{6}$ - Zusammenhänge. Die vielfältige Interdependenz innerhalb des Umweltrechts erfordert bisweilen auch das bewußte Abstandnehmen von Fakten und ganzen Rechtsbereichen, um Klarheit für die Erkenntnis zu gewinnen. Beschränken möchte ich mich daher nur auf einen Teilbereich des Umweltrechts, auf das Naturschutzrecht, damit also gewissermaßen auf einen Aspekt aus den Kernbereich dessen, was sich als das Recht der natürlichen Lebensbedingungen umschreiben läßt.

Bereits der Name unserer Zeitschrift legt es nahe, sich des weiteren auf Übersee zu beschränken, ${ }^{7}$ auch wenn VERFASSUNG UND RECHT IN ÜBERSEE selbst nie wirklich Wert auf den "Salzwassertest" gelegt hat. ${ }^{8}$ Zugleich erübrigt der Name der Zeitschrift jede weitere Begründung und nimmt mir auch die Last, den diffusen Begriff "Übersee" genauer zu definieren, als es in 117 Ausgaben bisher gelungen ist. ${ }^{9}$

So sehr gerade derzeit der erneute Versuch, den Konflikt zwischen Nutzung und Schutz im deutschen Naturschutzrecht mit einer Novelle zum Bundesnaturschutzgesetz zu bewältigen, Diskussionsstoff bietet und so sehr auch auf europarechtlicher Ebene erst kürzlich wieder Vertragsverletzungsverfahren gegen die Bundesrepublik Deutschland wegen der Nichtumsetzung europarechtlicher Naturschutzrichtlinien Aufsehen erregt haben: Anders als in Europa, wo das Bestreben, Nutzung und Schutz einander näher zu bringen, erste Früchte

Zum Gesichtspunkt der Anthropozentrik im Umweltvölkerrecht vgl. nur Patricia Birnie / Alan E. Boyle, International Law and the Environment, Oxford 1992, S. 193.

Umweltrecht läßt sich - in größerem Maße als sonstiges Recht - als bloße Reaktion auf veränderte oder anders wahrgenommene tatsächliche Verhältnisse verstehen; vgl. dazu Fn. 77.

Da im folgenden ein Schwerpunkt auf der Erörterung des geltenden universellen Völkervertragsrechts liegt, besitzen die Ausführungen insoweit auch generelle Bedeutung.

Auch Regionen diesseits der "See" wurden immer wieder berücksichtigt: Vgl. z.B. die Beiträge von Alexander Blankenagel, Verfassungsgebung im GUS-Land, VRÜ 27 (1994), S. 5, und von Christian Rumpf, Das Nationalismusprinzip in der türkischen Verfassung, VRÜ 25 (1992), S. 407, oder kürzlich - zur Verfassungsvergleichung mit der Türkei - Sylvia Tellenbach, VRÜ 29 (1996), S. 375.

Vgl. zu einer ersten Ortsbestimmung nur Herbert Krüger, Verfassung und Recht in Übersee. Das Programm, VRÜ 1 (1968), S. 6, der die neuen politischen Einheiten in Übersee durch eine vergleichbare Entschlossenheit zur Modernisierung durch Revolution charakterisiert und gerade in der damit umschriebenen nicht nur geographischen Vielfalt die Grundlage für eine fruchtbare Befassung mit der Dialektik von Gleichheit und Verschiedenheit sieht. Die übrige Welt, neben Nordamerika z.B. auch Australien und Japan, nicht zuletzt aber Europa, rückt damit an den Rand, jedoch als tertium comparationis nicht aus dem Blickfeld. 
trägt, ${ }^{10}$ stellt sich der angesprochene Konflikt zwischen Umwelt und Entwicklung gerade in Übersee in weit existenziellerer Weise. Als Beleg mag ein Beispiel genügen, das den Bericht der nach ihrer Vorsitzenden kurz "Brundtland-Kommission" genannten UN-Kommission für Umwelt und Entwicklung aus dem Jahre 1987 illustriert:

In den Wäldern im Westen Ecuadors schätzte man im Jahre 1960 eine Zahl von ca. 200.000 Tier- und überwiegend endemischen Pflanzenarten. In der Zeit bis 1982 wurde der überwiegende Teil dieser Wälder zugunsten von Bananen-Plantagen, Öl-Brunnen und Siedlungen zerstört. Man schätzt den Verlust von über 50.000 Arten in nur 22 Jahren. ${ }^{11}$ Selbst wer die Artenvielfalt als eine noch zu unklare Kategorie, nicht als einen schutzbedürftigen Wert an sich ansieht, müßte von den Folgen des Verlustes an Tieren und Pflanzen, an Lebensräumen, die von diesen mitbestimmt werden, sowie den wirtschaftlichen, sozialen und kulturellen Folgen für die Bevölkerung betroffen sein. ${ }^{12}$

Noch zwei weitere Bemerkung zu Übersee: Aus der Sicht des Naturschutzes sind die in Übersee noch vorhandenen Ressourcen beeindruckend. Mindestens ebenso beeindruckend sind die immer neuen Schreckensmeldungen erheblicher und nachhaltiger Zerstörungen der Natur. Das Augenmerk auf Übersee zu lenken, soll jedoch nicht den Eindruck erwecken, Europa seien solche Probleme fremd. Vielmehr sind in Europa lediglich weit weniger Naturräume verblieben, die noch für einen sinnvollen Schutz in Betracht kommen.

Die Blickrichtung auf Übersee soll im übrigen nicht den Eindruck erwecken, die Problematik von Umwelt und Entwicklung oder das zu ihrer Lösung herangezogene Konzept des sustainable development beschränkten sich auf die Staaten des Südens, nachhaltige Entwicklung sei möglicherweise im Kontext von Entwicklungsvölkerrecht zu sehen. ${ }^{13}$

Bei aller Unklarheit über Umfang und Nutzen des Erreichten wurden z.B. beachtliche Schritte in Hinblick auf die Identifizierung von Lücken bestehender Schutzkonzepte unternommen: vgl. zu einem besonders komplexen Beispiel der Verschränkung von Nutzung und Schutz großflächiger grenzüberschreitender Feuchtgebiete Karel van der Zwiep / Chris Backes, Integrated System for Conservation of Marine Environments, Baden-Baden 1994, S. 71 f., sowie zu ermutigenden Beispielen nachhaltiger Ressourcennutzung gerade auch im Norden Ernst Ulrich von Weizsäcker / Armory B Lovins / L. Hunter Lovins, Faktor Vier. Bericht an den Club of Rome, München 1995, S. $31,241$.

World Commission on Environment and Development, Our Common Future, Oxford 1987, S. 149, mit Hinweis auf A.H. Gentry, Patterns of Neotropical Plant Species Diversity, Evolutionary Biology, Vol. 15 (1982), S. 1. Eingehend hierzu E. O. Wilson, Der gegenwärtige Stand der biologischen Vielfalt, in: ders. (Hrsg.), Ende der biologischen Vielfalt?, Heidelberg 1992, S. 19.

Eine Aufstellung von Gründen für den Artenschwund findet sich bei Philippe Sands, Principles of international environmental law, Vol. I, Manchester 1995, S. 369.

13

Zum Entwicklungsvölkerrecht vgl. Guido Odendahl, Das Recht auf Entwicklung - The Right to Development, Aachen 1997, S. 47 und 83. 
Es muß es hier genügen, mit einer auf rechtliche Aspekte beschränkten Betrachtung den Geltungsanspruch des positiven Rechts zu prüfen und Gedanken über eine künftige Rechtsentwicklung anzustellen. Eine empirische Evaluierung der Effizienz rechtlicher Regelungen kann hier ebensowenig geleistet werden, wie eine wirkliche Lösung des Problems. Denn das Recht ist nur ein, möglicherweise nicht einmal ein besonders geeigneter Ansatz zur Lösung der sich im Spannungsfeld von Umwelt und Entwicklung ergebenden Probleme. Die - noch enger - auf das Naturschutzrecht beschränkte Sichtweise sei hier dennoch erlaubt, da das internationale Naturschutzrecht, neben verfahrensrechtlichen Konzepten zur Fortentwicklung von Rechtsetzung ${ }^{14}$ und Rechtsdurchsetzung im Völkerrecht, ${ }^{15}$ im Vordergrund der Diskussion um die Umsetzung nachhaltiger Entwicklung im Recht steht. $^{16}$

Die globale Problematik von Naturschutz und nachhaltiger Entwicklung in Übersee bietet sich für eine völkerrechtliche Betrachtung an. Möglicherweise ergibt sich daraus für zukünftige Ausgaben dieser Zeitschrift auch eine Auseinandersetzung über die Umsetzung im innerstaatlichen Recht einzelner Staaten Afrikas, ${ }^{17}$ Asiens ${ }^{18}$ oder Lateinamerikas. ${ }^{19}$

Zur Fortentwicklung der Rechtsetzung auf internationalen Konferenzen wie der Rio-Konferenz in einem "Prozeß" der eine Vielzahl neuer Akteure einbezieht und weit über den Rahmen einer einzigen Konferenz hinausgeht Bertram I. Spector / Gunnar Sjöstedt / I. William Zartman (eds.), Negotiating International Regimes, London 1994.

Vgl. hierzu den Bericht von Ulrich Beyerlin und Thilo Marauhn, Rechtsetzung und Rechtsdurchsetzung im Umweltvölkerrecht nach der Rio-Konferenz 1992, Berichte des Umweltbundesamtes 3/97, Berlin 1997, sowie die Beiträge des Workshop on Institution-Building in International Environmental Law, ZaöRV 56 (1996), S. 601; vgl. auch Alexandre Kiss, The implications of global change for the international legal system, in: Edith Brown Weiss (ed.), environmental change and international law, Tokyo 1992, S. 315.

Vgl. Birnie / Boyle, International Law and the Environment (Fn. 5), S. 6; zum Ausgangspunkt Naturschutz für die Diskussion um die Nachhaltigkeit von Weizsäcker et al., Faktor Vier (Fn. 10), S. 241 .

Vgl. zum bisher geltenden Recht bereits Costa R. Mahalu, Environmental Degradation and the Law in Tanzania, VRÜ 22 (1989), S. 460.

Vgl. bisher Joachim Schabacker, Chinesisches Umweltrecht, VRÜ 29 (1996), S. 31; Emmanuel Opoku Awuku, The Right to a Clean Environment: Lessons form India and Tanzania, VRÜ 27 (1994), S. 516.

Vgl. bisher Brigitte Lhoëst, The Legal and Administrative Context of Environmental Policy in Mexico City, VRÜ 27 (1994), S. 141; Günther Maihold, Umweltprobleme als Thema der Außenpolitik: Mexiko - USA, VRÜ 22 (1989), S. 392. 


\section{Thesen}

Einige Thesen mögen vorab das Verhältnis von völkerrechtlichem Naturschutz zu nachhaltiger Entwicklung in Übersee verdeutlichen.

1. Der völkerrechtliche Naturschutz ist älter als das (man muß wohl formulieren: sonstige) Umweltvölkerrecht. Das geltende Naturschutzrecht ist aber nicht nur ein erprobter und effektiver Bereich des Umweltvölkerrechts. In seiner Entwicklung hat das Naturschutzrecht jeweils auch sehr schnell auf neue wissenschaftliche Erkenntnisse und Tendenzen mit der Normierung neuer Schutzgüter und Schutzziele reagiert.

2. Der Begriff "nachhaltige Entwicklung" - oder sustainable development, beide Begriffe sollen im folgenden synonym verwendet werden ${ }^{20}$ - wurde im Jahre 1980 geprägt. Er kennzeichnet, wie sich auch anhand des völkerrechtlichen Naturschutzes nachzeichnen läßt, den Weg des Umweltvölkerrechts von der Stockholmer Umweltkonferenz 1972 zur Weltgipfelkonferenz von Rio 1992.

3. Das in der Rio-Deklaration von 1992 enthaltene Konzept eines sustainable development versteht sich als Leitbild für die zukünftige Entwicklungs- und Umweltpolitik aller Staaten der einen Welt. Es weist damit weit über den Stand heutiger Erkenntnisse und heute geltendes Recht hinaus. Allerdings ist es weder völlig neu noch ist es auf alle Bereiche gleichermaßen anwendbar.

4. Im universellen völkerrechtlichen Naturschutz hat das Konzept - wenn auch ohne ausdrückliche Nennung - in der Konvention über die biologische Vielfalt von 1992 seinen ersten vertraglichen Niederschlag gefunden, bedarf aber der weiteren Konkretisierung im Völkervertragsrecht. Seinem Inhalt nach geht es weit über die in der Konvention ausdrücklich formulierte Pflicht zur nachhaltigen Nutzung (sustainable use) hinaus.

5. Weder das in der Rio-Deklaration noch das in der Konvention über die biologische Vielfalt enthaltene Konzept eines sustainable development führen zu einem allgemeinen Entwicklungsvorbehalt im völkerrechtlichen Naturschutz.

6. Das Konzept des sustainable development im Übereinkommen über die biologische Vielfalt unterscheidet zwischen Erhaltung der biologischen Vielfalt, der nachhaltigen Nutzung von Bestandteilen der biologischen Vielfalt und dem allgemeinen Schutz vor Beeinträchtigungen durch sonstige Nutzungen. In keinem dieser Bereiche wirkt sich die

Da hier nicht die Umsetzung des völkerrechtlichen Konzepts des "sustainable development" in das innerstaatliche Recht thematisiert wird und es damit auf die Reichweite des im deutschen Forstrecht entwickelten Begriffes der "Nachhaltigkeit" nicht ankommt (vgl. hierzu Meinhard Schröder, Nachhaltigkeit als Ziel und Maßstab des deutschen Umweltrechts, Wirtschaft und Verwaltung 1995, S. 67), steht der - ansonsten ungenauen - synonymen Verwendung des Terminus "nachhaltige Entwicklung" nichts im Wege. 
Bezugnahme auf das Verhältnis von Umwelt und Entwicklung relativierend aus. Auch im Verhältnis zu anderen Übereinkommen zum Schutz der Natur ergeben sich keine Abschwächungen, allenfalls Verschärfungen des Schutzes.

7. Die Formel "as far as possible and as appropriate" (soweit möglich und sofern angebracht) führt nicht zu einer abgestuften Verpflichtung, einem doppelten völkerrechtlichen Standard zugunsten des Südens und zu Lasten des Nordens, sondern resultiert in einer entsprechenden Verpflichtung aller Staaten nach ihren jeweiligen Möglichkeiten. Eine wirkliche Sonderstellung wird entwickelten Ländern lediglich in Hinblick auf den Technologietransfer und ihre Finanzierungsverantwortung auferlegt - eine weiterreichende Differenzierung findet nicht statt.

8. Im überseeischen regionalen Völkervertragsrecht wurde mit dem Protokoll über Schutzgebiete und wildwachsende Fauna und Flora in Ostafrika und dem - ebenfalls aus dem Jahre 1985 stammenden - ASEAN-Übereinkommen noch früher als auf universeller Ebene mit der vertraglichen Umsetzung des Konzepts der nachhaltigen Entwicklung begonnen. Hieraus und aus der im übrigen beachtlichen Dichte völkervertraglicher Regelungen zum Naturschutz, die zusammen genommen weitere Aspekte nachhaltiger Entwicklung regeln, ist eine positive Tendenz für die weitere Umsetzung des Konzepts auf regionaler völkerrechtlicher Ebene, möglicherweise auch für die innerstaatliche Ebene, zu entnehmen.

\section{Die Einbeziehung Konzepts nachhaltiger Entwicklung in den völkerrechtlichen Naturschutz}

Der völkerrechtliche Naturschutz ist älter als das sonstige Umweltvölkerrecht. Er wird - in seiner Ausprägung als Artenschutzrecht ${ }^{21}$ - teils zusammen mit dem Gewässerschutz recht $^{22}$ als erste Stufe in der Entwicklung zu einem allgemeinen Umweltvölkerrecht gesehen. 23

Zu den Anfängen des Schutzes natürlicher lebender Ressourcen vor Ausbeutung Sands, Principles (Fn. 12), S. 25, und Birnie / Boyle, International Law and the Environment (Fn.5), S. 421.

Vgl. Philip Kunig, Nachbarrechtliche Staatenverpflichtungen bei Gefährdungen und Schädigungen der Umwelt, in: Umweltschutz im Völkerrecht und Kollisionsrecht, Berichte der Deutschen Gesellschaft für Völkerrecht, Heft 32, Heidelberg, 1992, S. 11.

Vgl. Albrecht Randelzhofer, Umweltschutz im Völkerrecht. Grundstrukturen und Prinzipien, JURA 1992, S. 3. 
Die zweite Stufe bildet das Umweltnachbarrecht, das aufgrund vielfältiger Staatenpraxis in den 30er Jahren dieses Jahrhunderts ${ }^{24}$ völkergewohnheitsrechtliche Regeln entstehen ließ. Eine zentrale Regel des völkerrechtlichen Nachbarrechts ist das Verbot, auf dem eigenen Staatsgebiet Handlungen vorzunehmen, die nicht unerhebliche, unübliche Schädigungen auf dem Gebiet von Nachbarstaaten hervorrufen. ${ }^{25}$ Dieses Verbot erheblicher Schadenszufügung wurde, zusammen mit der Bekräftigung der souveränen Rechte der Staaten, die eigenen Ressourcen nach Maßgabe der eigenen Umweltpolitik zu nutzen, von Prinzip 21 der Stockholmer Deklaration ${ }^{26}$ über die Umwelt des Menschen vom 16. Juni 1972 aufgegriffen und fortgebildet. ${ }^{27}$ Die in Ergänzung des Völkergewohnheitsrechts geschlossenen nachbarrechtlichen Verträge zwischen Anrainerstaaten betreffen überwiegend die Nutzung von Ressourcen aquatischer Systeme, insbesondere von Flüssen und Seen. ${ }^{28}$

Das Umweltvölkerrecht als solches hat sich erst auf einer dritten Stufe entwickelt: Seine ersten Ausprägungen fand es in den Genfer Seerechtsübereinkommen ${ }^{29}$, in Regeln über den Schutz der Antarktis ${ }^{30}$ und die grenzüberschreitende Luftverunreinigung. ${ }^{31}$ Es widmet sich überwiegend globalen Umweltproblemen. ${ }^{32}$

\section{Universelles völkerrechtliches Naturschutzrecht}

Demgegenüber konzentrierte sich der völkerrechtliche Naturschutz zunächst auf regionale Probleme: Die erste multilaterale Übereinkunft zum Artenschutz aus dem Jahre 1902, die heute noch in Kraft ist, widmet sich dem Schutz der für die Landwirtschaft nützlichen

24 Hier wird, wenn auch in Hinblick auf die Staatenpraxis und das angewandte Recht nicht unumstritten, auf den Schiedsspruch im sog. Trail-Smelter-Fall verwiesen, vgl. 3 UNRIAA 1905; Text auch in: Philippe Sands / Richard Tarasofsky / Mary Weiss (eds.), Documents in international environmental law, Band IIA, Manchester 1994, S. 91.

Vgl. Randelzhofer, Umweltschutz (Fn. 23), JURA 1992, S. 6.

Sands et al. (eds.), Documents (Fn. 24), S. 14.

27

Vgl. Kunig, Nachbarrechtliche Staatenverpflichtungen (Fn. 22), S. 12.

Kunig, Nachbarrechtliche Staatenverpflichtungen (Fn. 22), S. 28 f.; vgl. auch Eberhard Klein, Umweltschutz im völkerrechtlichen Nachbarrecht, Berlin 1976.

Vgl. nur Renate Platzöder/Horst Grunenberg, Internationales Seerecht, München 1990, S. 684.

Vgl. Antarktis-Vertrag vom 1.12.1959, BGBl. 1978 II S. 1518, engl. Text in: Sands et al. (eds.), Documents (Fn 24), S. 1583.

31 Übereinkommen über weiträumige grenzüberschreitende Luftverunreinigung vom 13.11.1979, Sands et al. (eds.), Documents (Fn 24), S. 121.

32

Vgl. Randelzhofer, Umweltschutz (Fn. 23), JURA 1992, S. 6; Kunig, Nachbarrechtliche Staatenverpflichtungen (Fn. 22), S. 12. 
Vögel. ${ }^{33}$ Kennzeichnend für das frühe völkerrechtliche Naturschutzrecht ist der am Nutzen - oder der Nutzung - ausgerichtete Schutz. ${ }^{34}$

Auch eine Zahl weiterer universeller völkerrechtlicher Verträge zum Naturschutz bestätigen diesen Befund: Zu nennen sind das Übereinkommen über den Schutz wilder Tiere und Pflanzen vom 8 . November $1933^{35}$, das Internationale Pflanzenschutzabkommen vom 6. Dezember $1951^{36}$ und das Übereinkommen zur Regelung des Walfangs vom 2. Dezember $1946^{37}$. Insbesondere der Walfang hält wegen seiner engen Verknüpfung von wirtschaftlicher Nutzung und Schutz bis auf den heutigen Tag die öffentliche Diskussion in Atem. $^{38}$

a)

\section{Ramsar-Übereinkommen}

Eine wesentliche Fortentwicklung ergab sich aus dem Ramsar-Übereinkommen von $1971,{ }^{39}$ das dem Schutz von Feuchtgebieten, ${ }^{40}$ insbesondere als Lebensraum für Wasserund Watvögel, von internationaler Bedeutung dient. Es folgt der Erkenntnis in die grundlegende ökologische Bedeutung von Feuchtgebieten als Regulatoren für den Wasserhaushalt und als Lebensraum für eine besondere Pflanzen- und Tierwelt. ${ }^{41}$ So tritt der Biotop-

33

34

35

36

37

38

39

40

41

Übereinkunft zum Schutz der für die Landwirtschaft nützlichen Vögel vom 19.03.1902, RGBl. 1906 II S. 89.

Gleiches gilt für Fischereiübereinkommen, vgl. Sands, Principles (Fn. 12), S. 26.

Convention Relative to the Preservation of Fauna and Flora in their Natural State, London (1933); Text in: Alexandre Charles Kiss (ed.), Selected Multilateral Treaties in the Field of the Environment, United Nations Environment Programme, Nairobi 1983, S. 3, 57.

BGBl. 1956 II S. 947; Kiss (ed.), Selected Multilateral Treaties (Fn. 35), S. 7, 90.

Neufassung des ursprünglichen Übereinkommens vom 24.09.1931, Protokoll vom 19.11.1956, BGBl. 1982 II S. 558; Sands et al. (eds.), Documents (Fn. 24), S. 701; vgl. auch Philip Kunig, Walfang, in: Otto Kimminich / Heinrich von Lersner / Peter-Christoph Storm, Handbuch des Umweltrechts, Band 2, 2. Auflage, Berlin 1994, Sp. 2710.

$\mathrm{Zu}$ einer umfassenden Darstellung der rechtlichen Problematik mit Bezügen zu der naturwissenschaftlichen Diskussion vgl. Gregory Rose / Saundra Crane, The evolution of international whaling law, in: Philippe Sands (ed.), Greening International Law, London 1993, S. 159.

Übereinkommen über Feuchtgebiete, insbesondere als Lebensraum für Wasser- und Watvögel vom 11.02.1971 (Ramsar-Übereinkommen), BGBl. 1976 II S. 1265; Sands et al. (eds.), Documents (Fn. 24), S. 737.

Das Ramsar-Übereinkommen ist bisher das einzige, das eine spezielle Art von Ökosystemen, hier Feuchtgebiete, schützt; vgl. Daniel Navid, The Ramsar Convention today, in: IUCN (ed.), Legal Aspects of the Conservation of Wetlands, Gland 1991, S. 36; ebenso Sands, Principles (Fn. 12), S. 404.

Vgl. Simon Lyster, International Wildlife Law, Cambridge 1985, S. 183. 
flächenschutz ${ }^{42}$ neben das Artenschutzrecht und löst den Schutzaspekt von dem wirtschaftlichen Nutzen; seither kann von einem umfassenden Naturschutzrecht gesprochen werden.

Damit waren, als die Stockholmer Konferenz der Vereinten Nationen über die Umwelt des Menschen am 16. Juni 1972 Prinzipien für einen umfassenden und universellen Umweltschutz formulierte, bereits der Biotopflächen- und Lebensraumschutz als zweite Säule ${ }^{43}$ neben den völkervertraglich in verschiedenen Instrumenten verankerten Artenschutz getreten. Wenig später folgten weitere völkerrechtliche Instrumente zum Ausbau des Naturschutzrechts.

\section{b) Übereinkommen zum Schutz des Kultur- und Naturerbes der Welt}

Das Übereinkommen zum Schutz des Kultur- und Naturerbes der Welt vom 23. November $1972^{44}$ unterstellt einerseits Stätten des Naturerbes (Naturgebilde, Naturstätten und genau abgegrenzte Lebensräume für bedrohte Tier- und Pflanzenarten), andererseits solche des Kulturerbes besonderem Schutz. Ebenso wie bei dem Ramsar-Übereinkommen werden die gemeldeten Schutzgebiete in eine Liste eingetragen. Die Vertragsparteien erkennen an, daß die in der Liste enthaltenen Schutzobjekte als "Welterbe der ganzen Menschheit" erhalten und an künftige Generationen weitergegeben werden müssen (Art. 4 I und 6 I). Der Schutz der in der Liste aufgeführten Objekte kann bei Bedarf aus einem Fonds, der aus Beiträgen der Vertragsparteien gespeist wird, mitfinanziert werden. Die Konvention greift den Grundsatz der Verantwortung auch für künftige Generationen ${ }^{45}$ auf, ohne aber der Nachwelt eine eigene - im Wege der Prozeßstandschaft vorzeitig geltend zu machende - Rechtsstellung zu verleihen. ${ }^{46}$ Damit kommt ein Gedanke zum Ausdruck, der auf der Konferrenz von Rio de Janeiro 20 Jahre später als common concern of humankind die Diskussion mitbestimmt hat.

Nicht nur sprachlich, sondern vor allem in Bezug auf den Schutz künftiger Generationen hat diesem Grundsatz, der in Rio de Janeiro in das Übereinkommen über biologische Vielfalt Eingang gefunden hat (Abs. 3 der Präambel), der ältere Grundsatz des common heri-

Zu der auch zahlenmäßigen Bedeutung von Schutzgebieten auf globaler Ebene vgl. Sands, Principles (Fn. 12), S. 372.

Das Inkrafttreten des Ramsar-Übereinkommens sollte jedoch noch 4 Jahre auf sich warten lassen. BGBl. 1977 II S. 215; Sands et al. (eds.), Documents (Fn. 24), S. 746.

45

Vgl. zur Verantwortung für zukünftige Generationen, Edith Brown Weiss, Conservation and Equity between Generation, in: Thomas Buergenthal (ed.), Contemporary Issues in International Law. Essays in Honour of Louis B. Sohn, Kehl 1984, S. 245.

$\mathrm{Zu}$ einer solchen Möglichkeit in Zweifelsfällen vgl. die Überlegungen von Sands, Principles (Fn. 12), S. 200. 
tage of mankind ${ }^{47}$ Pate gestanden. Allerdings berücksichtigt dieser nunmehr in Art. 136 des Seerechtsübereinkommens der Vereinten Nationen vom 10. Dezember $1982^{48}$ verankerte Verteilungsgrundsatz zur Nutzung des gemeinsamen Raumes und der Ressourcen auf dem Tiefseeboden den Schutz der Meeresumwelt (Art. 145) und die Entwicklung geographisch benachteiligter Staaten (Art. 148) nur am Rande.

\section{c) Washingtoner Artenschutzübereinkommen}

Mit dem Übereinkommen über den internationalen Handel mit gefährdeten Arten freilebender Tiere und Pflanzen (Washingtoner Artenschutzübereinkommen - CITES) vom 3. März $1973^{49}$ wurde ein sehr wirksames Artenschutzinstrument geschaffen, das durch das Verbot des internationalen Handels mit genau aufgeführten Arten und ihrer Teile, durch Ausfuhr und Einfuhr-Bescheinigungen den Hintergrund für die Jagd auf seltene Pflanzen und Tiere - den legalen Markt - ausgetrocknet hat. Seine Erfolgsgeschichte kann nicht nur durch die überzeugende Zahl von über Hundert Vertragsparteien, sondern auch durch Schutzerfolge belegt werden. Wurde noch im Jahre 1990 angenommen, daß der afrikanische Elefant bis zum Jahr 2000 ausgerottet sein werde, ${ }^{50}$ weil der Schutzmechanismus des CITES nicht greife, obwohl im Jahre 1989 durch die Hochstufung in Anhang I des CITES dessen strengster Mechanismus aktiviert worden sei, ${ }^{51}$ so wird heute bereits seine Überpopulation beklagt, die kürzlich zu einer Rückstufung in Anhang II geführt hat. Das Beispiel belegt nicht nur die Wirksamkeit ${ }^{52}$ der Instrumente des Washingtoner Artenschutzübereinkommens, sondern wirft zugleich die grundsätzliche Problematik von Artenschutz in Räumen auf, in denen der Mensch durch sein Eingreifen - und durch sonstige Kulturpflege - das ökologische Gleichgewicht beeinträchtigt.

Vgl. hierzu Arvid Pardo, The Common Heritage, Malta 1975; Carl August Fleischer, The International Concern for the Environment: The Concept of Common Heritage, in: Michael Bothe (ed.), Trends in Environmental Policy and Law, Berlin 1980, S. 321; Rüdiger Wolfrum, The Principle of the Common Heritage of Mankind, ZaöRV 43 (1983), S. 312.

Montego Bay Convention, BGBl. 1994 II S. 1799; Platzöder / Grunenberg, Seerecht (Fn. 29), S. $1,295$.

BGBl. 1975 II S. 773; Sands et al. (eds.), Documents (Fn. 24), S. 765.

Michael J. Glennon, Has International Law Failed the Elephant? AJIL 84 (1990), S. 1.

Vgl. nur die Kritik am CITES bei Harald Hohmann, Präventive Rechtspflichten und -prinzipien des modernen Umweltvölkerrechts, Berlin 1992, S. 329 f.

$\mathrm{Zu}$ derselben Einschätzung in Hinblick auf dieses Beispiel gelangen auch Alexandre Kiss / Dinah Shelton, International Environmental Law, New York, 1994 Supplement, S. 262. 
Das Übereinkommen zur Erhaltung der wandernden wildlebenden Tierarten (Bonner Übereinkommen) vom 23. Juni $1979^{53}$ schützt ebenso wie das Ramsar-Übereinkommen in erster Linie wandernde Tierarten. Anders als jenes verpflichtet das Bonner Übereinkommen zwar nicht zur Einrichtung von Schutzgebieten, betrifft aber mit dem Schutz der Lebensräume wandernder Arten ebenfalls ein typischerweise durch das Völkerrecht zu regelndes Thema. Es wird konkretisiert durch Regionalübereinkommen für die sich in der ungünstigsten Erhaltungssituation befindenden Arten des Anhangs II. Bisher ${ }^{54}$ wurden Regionalübereinkommen zum Schutz von Seehunden, Kleinwalen und Fledermäusen ${ }^{55}$ geschaffen. Die Regionalabkommen betreffen jeweils das gesamte Verbreitungsgebiet der betreffenden Art.

\section{e) Übereinkommen über die biologische Vielfalt}

Den vorläufig letzten Schritt ${ }^{56}$ in Richtung auf einen möglichst umfassenden Naturschutz bildet das Übereinkommen über die biologische Vielfalt vom 5. Juni $1992 .^{57}$ Als Schutzgut wird erstmals die biologische Vielfalt ${ }^{58}$ als Eigenwert, sowie ihr Wert und der ihrer Bestandteile in ökologischer, genetischer, sozialer, wirtschaftlicher, wissenschaftlicher, erzieherischer, kultureller, freizeitbezogener und ästhetischer Hinsicht anerkannt. ${ }^{59}$ Die Einbeziehung der künftigen Generationen knüpft an die bereits erwähnten Instrumente und

BGBl. 1984 II S. 569; Sands et al. (eds.), Documents (Fn. 24), S. 822.

Zum Übereinkommen und Plänen für weitere Regionalübereinkommen vgl. Sands, Principles (Fn. 12), S. 446.

Abkommen zum Schutz der Seehunde im Wattenmeer vom 16.10.1990, BGBl. 1991 II S. 1307; Abk. zur Erhaltung der Kleinwale in der Nord- und Ostsee vom 31.03.1992, BGBl. 1993 II S. 1113; Abk. zur Erhaltung der Fledermäuse in Europa vom 4.12.1991, BGBl. 1993 II S. 1106; Sands et al. (eds.), Documents (Fn. 24), S.838.

Ausgeklammert bleiben an dieser Stelle die Schutzsyteme sonstiger überseeischer Räume wie der Antarktis; vgl. dazu nur Kiss / Shelton, International Environmental Law (Fn. 52), S. 88.

BGBl. 1993 II S. 1742; Sands et al. (eds.), Documents (Fn. 24), S. 845.

Sie wird definiert als: "Variabilität unter lebenden Organismen jeglicher Herkunft, darunter unter anderem Land-, Meeres- und sonstige aquatische Ökosysteme und die ökologischen Komplexe, zu denen sie gehören; dies umfaßt die Vielfalt innerhalb der Arten und zwischen den Arten und die Vielfalt der Ökosysteme" (Art. 2).

59

Zu den Mängeln des sonstigen Naturschutzrechts in Hinblick auf den Artenschwund vgl. Robin Churchill, The Contribution of Existing Agreements for the Conservation of Terrestrial Species and Habitats to the Maintenance of Biodiversity, in: Michael Bowman / Catherine Redgwell (eds.), International Law and the Conservation of Biological Diversity, London 1996, S. 89. Siehe auch die Rezension des Buches von Gudrun Henne in VRÜ 30 (1997), S. 97. 
Kodifikationen an. ${ }^{60}$ Abs. 3 der Präambel verweist darauf, daß die biologische Vielfalt ein gemeinsames Anliegen der Menschheit (common concern of humankind) ist. ${ }^{61}$

Ebenfalls verhältnismäßig neu in einer völkerrechtlichen Naturschutzkonvention ${ }^{62}$ ist die Einbeziehung der nachhaltigen Nutzung als zentralem Element des Konzepts der nachhaltigen Entwicklung, des sustainable development. Nachhaltige Nutzung (sustainable use) wird definiert als:

"die Nutzung von Bestandteilen der biologischen Vielfalt in einer Weise und einem Ausmaß, die nicht zum langfristigen Rückgang der biologischen Vielfalt führen, wodurch ihr Potential erhalten bleibt, die Bedürfnisse und Wünsche heutiger und künftiger Generationen zu erfüllen" (Art. 2).

Anders als der Begriff des sustainable use wird der Begriff des sustainable development nicht ausdrücklich im Vertragstext genannt. Das Übereinkommen enthält jedoch eine Vielzahl von Bestimmungen, die ihrerseits weitgehend den rechtlichen Elementen des sustainable development-Konzepts entsprechen, das von der Expertengruppe der Weltkommission für Umwelt und Entwicklung im Juni $1986^{63}$ entwickelt ${ }^{64}$ und in der Rio-Deklaration über Umwelt und Entwicklung vom 16. Juni $1992^{65}$ formuliert wurde.

Von zentraler Bedeutung sind danach insbesondere der Schutz zukünftiger Generationen (inter-generational equity), der Schutz der biologischen Vielfalt verknüpft mit nachhaltiger Nutzung (sustainable use) von Ressourcen, der Grundsatz der Rücksichtnahme auf andere Staaten (intragenerational equity) und die Einbeziehung des Schutzes der biologischen Vielfalt in die innerstaatliche Planung (principle of integration). ${ }^{66}$ Damit setzt das Über-

60

61

62

63

64

65

66

Zur der Verantwortung für zukünftige Generationen in anderen Verträgen vgl. Sands, Principles (Fn. 12), S. 200.

Die Formulierung "common heritage of mankind" (vgl. oben bei Fn. 47) wurde wegen Bedenken bezüglich der Souveränität der betroffenen Staaten über ihre Ressourcen (vgl. Art. 3) nicht übernommen; vgl. Kiss / Shelton, International Environmental Law (Fn. 52), S. 103.

In Art. 1 Abs. 1 des ASEAN-Naturschutzübereinkommens von 1985 (vgl. unten Fn. 105) findet sich bereits als Grundprinzip die Formulierung: "to ensure the sustainable utilization of harvested resources under their jurisdiction in accordance with scientific principles and with a view to attaining the goal of sustainable development."

Experts Group on Environmental Law of the World Commission on Environment and Development, Environmental Protection and Sustainable Development. Legal Principles and Recommendations, London 1986, S. 9 ff.

Der Vorschlag ist rechtlich unverbindlich: Vgl. Birnie / Boyle, International Law and the Environment (Fn. 5), S. 435.

Declaration on Environment and Development, Rio de Janeiro (1992), UN Doc. A/CONF. 151/5; Sands et al. (eds.), Documents (Fn. 24), S. 49

Vgl. auch Sands, Principles (Fn. 12), S. 199. 
einkommen über die biologische Vielfalt mit seinen Vorschriften zentrale Aspekte des Konzeptes der nachhaltigen Entwicklung der Rio-Deklaration um. ${ }^{67}$ Im Gegenzug erlaubt dies jedoch grundsätzlich nicht die Interpretation der Bestimmungen des Übereinkommens im Lichte der Deklaration. Hinsichtlich des geltenden Naturschutzrechts sind, da es sich um einen umstrittenen vertraglich vereinbarten Kompromiß handelt, vielmehr die einzelnen Bestimmungen des Übereinkommens maßgebend, auf die deshalb im einzelnen eingegangen werden soll.

Im Grundsatz stützt sich das Übereinkommen auf den nachbarrechtlichen Völkergewohnheitsrechtssatz, ${ }^{68}$ daß die Staaten das souveräne Recht besitzen, ihre eigenen Ressourcen gemäß ihrer eigenen Umweltpolitik zu nutzen sowie die Pflicht, dafür zu sorgen, daß durch Tätigkeiten, die innerhalb ihres Hoheitsbereichs oder unter ihrer Kontrolle ausgeübt werden, der Umwelt in anderen Staaten oder in Gebieten außerhalb der nationalen Hoheitsbereiche kein Schaden zugefügt wird (Art. 3). Dieser - wortgleich auch in Prinzip 21 der Stockholmer Deklaration über die Umwelt des Menschen von $1972^{69}$ enthaltene $^{70}$ Grundsatz wird jedoch durch die im Übereinkommen normierten Pflichten der Vertragsstaaten zum Schutz der Natur modifiziert und präzisiert.

Der Pflichtenkanon des Übereinkommens umfaßt zunächst eine planungsrechtliche Komponente: die Verpflichtung, auf nationaler Ebene Strategien, Pläne und Programme zur Erhaltung und nachhaltigen Nutzung der biologischen Vielfalt zu entwickeln und diese wiederum in andere sektorale Fachplanungen oder eine Gesamtplanung einzubeziehen (Art. 6). Diese Verpflichtung der Vertragsparteien steht allerdings unter dem Vorbehalt "entsprechend ihren besonderen Umständen und Möglichkeiten", womit nicht eine einseitige Abstufung von Pflichten gegenüber einzelnen Staaten oder Staatengruppen statuiert wird, insbesondere nicht eine Bevorzugung ökonomisch benachteiligter Staaten, ${ }^{71}$ sondern eine allgemeine Beschränkung auf die relativ zu der inneren Situation eines Staates beste-

Mit Hinweis auf Art. 1 des Übereinkommens über die biologische Vielfalt vgl. Elisabeth Dowdeswell, Sustainable Development: The Contribution to International Law, in: Winfried Lang (ed.), Sustainable Development and International Law, London 1995, S. 4.

Das völkergewohnheitsrechtliche Verbot enthält allerdings eine Beschränkung auf erhebliche Schadenszufügung. Zum Erfordernis der Erheblichkeit vgl. Kunig, Nachbarrechtliche Staatenverpflichtungen (Fn. 22), S. $19 \mathrm{f}$.

Stockholm Declaration of the United Nations Conference on the Human Environment; Sands et al. (eds.), Documents (Fn. 24), S.14. Skeptisch zum Inhalt J. G. Starke, Introduction to International Law, 10 ${ }^{\text {th }}$ Ed., London 1989, S. 406.

70

Erstmals findet sich damit Prinzip 21 in einer vertraglichen Vorschrift, vgl. Sands, Principles (Fn. 12), S. 382.

71

So aber Alan E. Boyle, The Rio Convention on Biological Diversity, in: Bowman / Redgwell (eds.), (Fn. 59), S. 44, mit Hinweis auf Prinzip 7 (common but differenciated responsibility) der Rio-Deklaration. 
henden strukturellen Möglichkeiten. Insbesondere setzt die Pflicht zur Einbeziehung der Naturschutzfachplanung in die sonstige sektorale Fachplanung oder eine Gesamtplanung die Existenz solcher Planungen in dem betreffenden Staat voraus.

Auf das Fehlen genauer Daten über die auf dem Staatsgebiet vorhandenen Bestandteile der biologischen Vielfalt, deren Zustand und über typische beeinträchtigende Tätigkeiten für seine Planung kann sich ein Staat dabei nur eingeschränkt berufen, da Art. 7 des Übereinkommens die Vertragsparteien selbst "soweit möglich und sofern angebracht" ("as far as possible and as appropriate") zu deren Bestimmung und Überwachung verpflichtet und somit den Staaten die Darlegungslast auch für solche Defizite aufbürdet. Durch umfangreiche Bestimmungs- und wissenschaftliche Überwachungsmaßnahmen sollen die in der Anlage I genauer umrissenen ${ }^{72}$ Ökosysteme und Lebensräume, Arten, Gemeinschaften, Genome und Gene zur Ermöglichung gezielter Schutzmaßnahmen für die Erhaltung und die nachhaltige Nutzung biologischer Ressourcen identifiziert und beobachtet werden. Neu ist - neben der im völkerrechtlichen Naturschutzrecht etablierten Unterteilung in Ökosysteme und Lebensräume (Biotopflächen- und Lebensraumschutz) sowie in Arten und Gemeinschaften (klassischer Artenschutz) - die Einbeziehung genetischer Ressourcen als Schutzgut. Das "genetische Material von tatsächlichem oder potentiellem Wert" (Art. 2) hat nicht nur für die Pharmaindustrie unschätzbaren wirtschaftlichen Nutzen, sondern besitzt erhebliche Bedeutung für die Entwicklung und Fortentwicklung in Bereichen der Nahrungs- und Futtermittelproduktion unter extremen klimatischen und geographischen Bedingungen.

Neben die planungsrechtlichen Instrumente stellt die Konvention ordnungsrechtliche Schutzmaßnahmen. ${ }^{73}$ Dem neuen Schutzgut der biologischen Vielfalt und dem gentechnischen und genetischen Teilaspekt entsprechend unterscheidet die Konvention zwischen der Schaffung von klassischen Schutzgebieten in der natürlichen Umgebung der Arten (sog. Insitu-Erhaltung, Art. 8) und, erstmals, der Erhaltung von Bestandteilen der biologischen Vielfalt außerhalb ihrer natürlichen Lebensräume (Ex-situ-Erhaltung, Art. 9), wobei auch gerade letztere nicht nur der Erhaltung, sondern auch der Wiedereinführung dieser Arten in ihre natürlichen Lebensräume dienen soll. Dem erweiterten klassischen Verständnis von Schutzgebieten, das Lebensräume und Ökosysteme einschließt, werden Tierparke und Genbanken an die Seite gestellt und damit die kulturelle Einwirkung des Menschen auf die Natur in ihren verschiedenen Erscheinungsformen für den Schutz, die Pflege und die Ent-

Listen zu schützender Ökosysteme an Stelle nur definitorischer Konkretisierungen wurden noch auf der Konferenz aus dem Vertragstext gestrichen; vgl. Gudrun Henne, Das Regime über die biologische Vielfalt von 1992, in: Thomas Gehring / Sebastian Oberthür (Hrsg.), Internationale Umweltregime, Opladen 1997, S. 192.

Zum Zusammenwirken beider vgl. Sands, Principles (Fn. 12), S. 382. 
wicklung von Bestandteilen der biologischen Vielfalt über die Generationen hinweg nutzbar gemacht.

Schließlich wird der Pflicht zur nachhaltigen Nutzung (sustainable use) eine eigene Vorschrift gewidmet. Als nützlich von den Vertragsstaaten identifizierte und von ihnen kontrollierte Bestandteile der natürlichen Vielfalt (biologische Ressourcen) sollen besondere Berücksichtigung bei der innerstaatlichen Entscheidungsfindung in Hinblick auf ihre Erhaltung und nachhaltige Nutzung finden und nachteilige Auswirkungen durch Maßnahmen im Zusammenhang mit der Nutzung verhindert werden (Art. 10). Von besonderer Bedeutung gerade auch für Übersee ist, daß die herkömmliche Nutzung biologischer Ressourcen im Einklang mit traditionellen Kulturverfahren, die mit den Erfordernissen der Erhaltung oder nachhaltigen Nutzung vereinbar sind, geschützt und gefördert werden soll. Ebenfalls wichtig vor allem in Übersee ist die Bedeutung, die die Konvention der Einbeziehung von ortsansässigen Bevölkerungsgruppen bei der Ausarbeitung und Durchführung von Abhilfemaßnahmen zubilligt (Art. 10 Buchst. d, e). Hier sollen sowohl Erfahrungen einbezogen als auch die Akzeptanz vor Ort für die Durchführung von Schutzmaßnahmen durch die Vermeidung von Planungen am grünen Tisch gestärkt werden. Für ein Verständnis, das vermeintlich moderner Ausbeutung von Ressourcen den Vorzug vor hergekommen Methoden und Verfahren gibt, ohne deren Auswirkungen auf die Natur zu untersuchen, läßt das Übereinkommen keinen Raum.

Neue Verpflichtungen betreffen wirtschaftlich und sozial verträgliche Anreize zur Erhaltung und nachhaltigen Nutzung von Bestandteilen der biologischen Vielfalt (Art. 11), die Aufklärung der Öffentlichkeit (Art. 13) und die Einführung von Umweltverträglichkeitsprüfungen für Vorhaben mit wahrscheinlich erheblichen Auswirkungen sowie die Schaffung von Notfallmaßnahmen zum Schutz der Natur vor akuten Gefahren durch Unfälle und Naturereignisse (Art. 14). Bei Forschung und Ausbildung von Personal soll den Bedürfnissen der Entwicklungsländer besonders Rechnung getragen werden (Art. 12). Auf internationaler Ebene werden die Erleichterung von Informationsaustausch (Art. 17) sowie technische und wirtschaftliche Zusammenarbeit (Art. 18) auf den angesprochenen Gebieten vereinbart. Denn die Akkumulation von Wissen und technologische Entwicklung können die Erschöpfung einer Ressource zwar hinauszögern, Nachhaltigkeit verlangt aber nach frühzeitiger Erkenntnis in die Endlichkeit der Ressource in der ganzen Welt, nach Zugang nach Grundsätzen der Billigkeit und Reorientierung der technischen Anstrengungen um den Druck auf die betreffenden Ressourcen zu lindern.

Einen neuen Regelungsbereich innerhalb der Konvention stellt der Zugang zu genetischen Ressourcen dar (Art. 15). ${ }^{74}$ Er ist gekoppelt mit dem Zugang zu und der Weitergabe von

74

Vgl. hierzu John Mugabe et al. (eds.), Access to Genetic Resources, Nairobi 1997; Rüdiger Wolfrum / Peter-Tobias Stoll, Der Zugang zu genetischen Ressourcen nach dem Übereinkommen 
Technologie einschließlich der Biotechnologie (Art. 16). Aus dem Blickwinkel des Naturschutzrechts handelt es sich bei diesen Regelungen nicht um einen Fremdkörper, sondern um Spezialvorschriften über die nachhaltige Nutzung biologischer und genetischer Ressourcen.

Die Konvention eröffnet damit nicht neue Tore für den Verbrauch und die Gefährdung von Natur durch Gentechnik, sondern beschreibt, regelt und beschränkt einen Aspekt der Naturnutzung, der in der jüngsten Vergangenheit in den Vordergrund gerückt ist. Zugleich liegt in der Erweiterung des Regimes ${ }^{75}$ zum Schutz der biologischen Vielfalt um die genetische Dimension ein Schlüssel für den Erfolg bei der Einigung auf eine Konvention über biologische Vielfalt auf der Konferenz für Umwelt und Entwicklung in Rio: Die Interessen von Nord und Süd, internationalen Konzernen und Umweltverbänden konnten auf der Konferenz zu einem gemeinsamen Nenner zusammengeführt und mit dem Übereinkommen über die biologische Vielfalt zu einem Paket zusammengeschnürt werden. ${ }^{76}$ Nicht zum ersten Mal ist die Einsicht in die Bedrohung einer nur begrenzt verfügbaren Ressource und die Erkenntnis ihrer Bedeutung für die Nutzung und den Schutz der Natur zum Motor für die Fortentwicklung des universellen Umweltvölkerrechts geworden. ${ }^{77}$ Zugleich belegen die Regelungen des Übereinkommens zur Gentechnik, daß das Naturschutzrecht nicht selten schnell auf neue wissenschaftliche Erkenntnisse und Tendenzen reagiert.

Dabei führte der Zwang zur Schaffung des gemeinsamen Nenners insgesamt nicht zu einer Aufweichung der Schutzstrukturen des völkervertragsrechtlich etablierten Naturschutzes durch Schaffung eines doppelten Standards von Rechtspflichten für Entwicklungsländer einerseits oder durch Anerkennung und Gestattung umfassender Nutzungsbefugnisse an Bestandteilen der Natur andererseits.

Differenzierte Rechtspflichten sieht das Übereinkommen ausschließlich in Hinblick auf den Finanzierungsmechanismus und den gentechnischen Technologietransfer vor. Neben den Grundsatz gleicher Verantwortung nach relativer Möglichkeit für die Erfüllung der eigenen Aufgaben (Art. 16 Abs. 1, Art. 20 Abs. 1) stellt es die Verpflichtung der entwickelten

über die biologische Vielfalt und dem deutschen Recht, Berlin 1996; zu informellen Schutzsystemen vgl. Kiss/Shelton, International Environmental Law (Fn. 52), S. 102.

Vgl. Thomas Gehring / Sebastian Oberthïr, Internationale Regime als Steuerungsinstrumente der Umweltpolitik, in: Gehring / Oberthür (Hrsg.), Internationale Umweltregime (Fn. 72), S. 9 ff.; vgl. auch Sebastian Oberthiir, Umweltschutz durch internationale Regime, Opladen 1997.

$\mathrm{Zu}$ den Reaktionen auf neue wissenschaftliche Erkenntnisse im Umweltvölkerrecht vgl. nur Edith Brown Weiss, Global environmental change and international law: The introductory framework, in: dies. (ed.), Environmental Change and International Law, Tokyo 1992, S. 4. Zum überwiegend reaktiven Charakter des Umweltrechts auch Jutta Brunée, Entwicklungen im Umweltvölkerrecht am Beispiel des sauren Regens und der Ozonschichtzerstörung, diss. iur., Mainz 1988, S. 380 f. 
Länder, die besondere, auch finanzielle Lage von Entwicklungsländern beim Technologietransfer (Art. 16 Abs. 2) und bei der Bereitstellung neuer und zusätzlicher Mittel ${ }^{78} \mathrm{zu}$ berücksichtigen (Art. 20 Abs. 2). ${ }^{79}$ Die Verantwortung der entwickelten Länder wird in engen Zusammenhang mit der wirksamen Erfüllung der Vertragsverpflichtungen durch die Entwicklungsländer gestellt (Art. 20 Abs. 4). Die Vorschrift ist Ausdruck des Prinzips der gemeinsamen aber differenzierten Verantwortung. ${ }^{80}$ Die sich in diesen Vorschriften widerspiegelnden Elemente des Rechts auf Entwicklung ${ }^{81}$ finden im übrigen keine gesonderte Erwähnung. Außerhalb der genannten Bestimmungen finden sich keine entsprechenden Ansätze, so daß die Vorschriften des Übereinkommens nicht die Einführung eines das gesamte Übereinkommen oder das materielle völkerrechtliche Naturschutzrecht erfassenden doppelten bzw. differenzierten Standards für entwickelte und Entwicklungsländer vorzeichnen.

Die Nutzung von Ressourcen, genauer: Bestandteilen der biologischen Vielfalt, wird durch das Konzept der nachhaltigen Entwicklung (sustainable development) bestimmt, das im Übereinkommen über die biologische Vielfalt in zweifacher Weise ausgeprägt ist: zum einen durch die Verschärfung und Ausdifferenzierung der naturschutzrechtlichen Schutzmechanismen zur Erhaltung der biologischen Vielfalt als Grundlage für eine nachhaltige Entwicklung und zum anderen durch die Sicherstellung der nachhaltigen Nutzung (sustainable use) als geeignet identifizierter biologischer Ressourcen.

Zum Schutz der biologischen Vielfalt für zukünftige Generationen verpflichtet das Übereinkommen zunächst zum wirksamen, großflächigen Schutz aller Bereiche in und außerhalb von Schutzgebieten, die für die Erhaltung der biologischen Vielfalt erforderlich sind (Art. 8 f.). Es knüpft insofern an die Entwicklung des Artenschutzes vom isolierten Schutz einzelner wertvoller Arten zum Schutz der Lebensräume zu schützender Arten bis hin zum Schutz bestimmter, generell als Lebensräume einer Vielfalt von Arten wichtiger Ökosysteme an, nicht ohne den Schutz einzelner bedrohter Arten durch innerstaatlich festzulegende Artenschutzlisten zu vernachlässigen (Art. 8 Buchst. k).

Erst der zweite Aspekt des Konzepts der nachhaltigen Entwicklung, der wegen seiner eigenständigen rechtlichen Bestimmungen getrennt vom ersten Aspekt zu sehen ist, regelt

Eine so weitgehende Finanzierungsverantwortung findet sich erstmals in einem Umweltschutzübereinkommen, vgl. Boyle, The Rio Convention on Biological Diversity, in: Bowman / Redgwell (eds.), (Fn. 59), S. 46.

79 Helmuth Beck, Die Differenzierung von Rechtspflichten in den Beziehungen zwischen Industrieund Entwicklungsländern, Frankfurt/Main 1994, S. 186 f.

80 Eingehend dazu Sands, Principles (Fn. 12), S. 217.

81 Zu den Inhalten vgl. Odendahl, Das Recht auf Entwicklung (Fn. 13); s. auch die Rezension in diesem Heft. 
die Nutzung im engeren Sinn. Hier knüpft das Übereinkommen an die herkömmliche Nutzung biologischer Ressourcen im Einklang mit traditionellen Kulturverfahren an (Art. 10 Buchst. c) und unterwirft diese und neue Nutzungen sowohl der Naturschutzplanung (Art. 6) als auch der Bestimmung und Überwachung (Art. 7) und sieht schließlich - mit Blick auf die zukünftige Entwicklung - die weitere Erforschung vor (Art. 12). Im Ergebnis werden die Bedürfnisse und Wünsche der gegenwärtigen Generation zur Nutzung von Ressourcen nicht unerheblich zugunsten künftiger Generationen beschränkt. Die nachhaltige Nutzung biologischer Ressourcen führt schon wegen der engen sachlichen Verknüpfung mit der Verschärfung der Schutzpflichten nicht zu einer Aufweichung des Schutzes der Natur zwischen den Vertragspartnern des Übereinkommens.

Auch im Verhältnis zu anderen Übereinkommen bewirken die Bestimmungen des Übereinkommens über die biologische Vielfalt zum sustainable use keine Schwächung des in diesen Verträgen - in seinen Ausprägungen als Artenschutzrecht und rechtlichem Schutz der Lebensräume und Biotopflächen - festgelegten Naturschutzstandards. Art. 22 stellt klar, daß die Rechte und Pflichten einer Vertragspartei aus bestehenden völkerrechtlichen Übereinkommen insoweit unberührt bleiben, als die Wahrnehmung der betreffenden Rechte und Pflichten die biologische Vielfalt nicht ernsthaft schädigen oder bedrohen würde. Das gilt auch für bestehendes regionales Völkervertragsrecht in Afrika, Lateinamerika und Asien. Eine Relativierung des bestehenden völkervertragsrechtlichen Naturschutzes durch das zum sustainable development gehörige Konzept der nachhaltigen Nutzung erfolgt demnach nicht.

Vielmehr ist eine Dynamisierung in Hinblick auf einige der oben angesprochenen anderen globalen Naturschutzübereinkommen nicht auszuschließen. Man denke nur an die Dynamik des Washingtoner Artenschutzabkommens ${ }^{82}$ oder die Schaffung weiterer Regionalabkommen zum Schutz der gesamten Verbreitungsgebiete bedrohter Arten nach der Bonner Konvention. ${ }^{83}$ Auch hier sind - als Folge der nach der Konferenz von Rio geschärften internationalen Aufmerksamkeit für den völkerrechtlichen Naturschutz - Fortentwicklungen, nicht Abschwächungen des Schutzniveaus zu erwarten.

Insgesamt gesehen trägt das Übereinkommen über die biologische Vielfalt überwiegend den Charakter eines Rahmenübereinkommens, ${ }^{84}$ das erst der Konkretisierung durch den

82

83

84

Zur Abgrenzung eines Rahmenübereinkommens für zukünftige Maßnahmen von einem Dachübereinkommen für bestehende Instrumente vgl. de Klemm / Shine, Biological Diversity (Fn. 2), S. 24. 
Folgeproze $\beta^{85}$ der UN-Konferenz über Umwelt und Entwicklung von Rio de Janeiro und durch Protokolle bedarf. ${ }^{86}$ Ist eine Aufweichung oder Relativierung des Naturschutzes durch das Übereinkommen über die biologische Vielfalt nicht erfolgt, so könnte dies daher noch durch die nachfolgenden Protokolle zum Übereinkommen geschehen. Eine solche Entwicklung des Nachfolgeprozesses zeichnet sich jedoch noch nicht ab. Vielmehr mündete das nahezu "instant" geschaffene internationale Bewußtsein ${ }^{87}$ für den Eigenwert der biologischen Vielfalt und ihrer engen Interdependenz mit verschiedenen Aspekten des Naturschutzes bereits unmittelbar in Anschluß an die Konferenz von 1992 zu verstärkter Erforschung der genauen naturwissenschaftlichen Zusammenhänge. ${ }^{88}$

Ungeachtet der noch erforderlichen Konkretisierungen und der noch unklaren Umsetzung der Konvention durch die Vertragsparteien hat das Übereinkommen über die biologische Vielfalt das universelle völkerrechtliche Naturschutzrecht mit der Einführung der biologischen Vielfalt als neuem Schutzgut und der Erweiterung des etablierten Maßnahmenkatalogs insbesondere um die Anerkennung und Nutzbarmachung der Ex-situErhaltung, die Einführung einer Umweltverträglichkeitsprüfung, die Beteiligung weiter Bevölkerungskreise auf allen Ebenen sowie weitreichende Forschungs- und Bestimmungspflichten einen erheblichen Schritt in Richtung auf einen umfassenden und damit langfristig wirksamen, nachhaltigen Schutz der Natur vorangebracht.

\section{f) Resümee zum universellen völkervertraglichen Naturschutz}

Die Entwicklung des völkervertraglichen Naturschutzes ist durch eine stetige Ausweitung des Kreises der Schutzgüter, eine größere Vielfalt von Instrumenten und die stärkere Bezugnahme auf den Ausgleich zwischen der Nutzung der Natur und ihrem Schutz gekennzeichnet. Das Konzept einer nachhaltigen Entwicklung - sustainable development - ist erstmals im Übereinkommen über die biologische Vielfalt von 1992 kodifiziert und prägt zugleich das gesamte Übereinkommen. Nachhaltige Entwicklung ist danach von einer Ausweitung des Schutzes auf die Bewahrung der biologischen Vielfalt für zukünftige Generationen durch Schutzmaßnahmen in situ und ex situ gekennzeichnet, durch Verant-

Dazu, insbesondere zu den Konferenzen von Jakarta (1995) und Buenos Aires (1996) vgl. Henne, Biologische Vielfalt (Fn. 72), S. 195.

86 Vgl. Henne, Biologische Vielfalt (Fn. 72), S. 194; Beck, Differenzierung von Rechtspflichten (Fn. 79), S. 186.

Die Erkenntnis in die Bedeutung und Bedrohung der biologischen Vielfalt ist weit älter: Vieles spricht dafür, die World Conservation Strategy von 1980 (oben Fn. 1) als Ausgangspunkt zu sehen. Vgl. Michael Bowman, The Nature, Development and Philosophical Foundations of the Biodiversity Concept in International Law, in: ders. / Redgwell (eds.), International Law (Fn. 59), S. 8.

Vgl. hierzu Vernon Heywood (ed.), Global Biodiversity Assessment, Cambridge 1995. 
wortung zwischen Nachbarstaaten und zwischen Generationen, durch Technologietransfer und einen neuen Finanzierungsmechanismus. Demgegenüber ist das Prinzip der gemeinsamen und differenzierten Verantwortlichkeit ein Element, das sich nur punktuell auswirkt, aber nicht zu einem generellen doppelten Standard führt.

Das völkerrechtliche Naturschutzrecht hat sich durch einen steten und progressiven Wandel von einem lückenhaften zu einem geschlossenen Schutzsystem entwickelt. Es hat dabei auf die globale Bedrohung des Artenverlustes mit der Einbeziehung des Schutzes biologischen Vielfalt einschließlich der genetischen Vielfalt, auf die vorhandenen Vollzugsdefizite mit der Schaffung bzw. Verankerung neuer Mechanismen (Finanzierungsmechanismus, Technologietransfer) reagiert. Zudem ist es mit seinen planerischen Komponenten und der Ausrichtung auf die Vorsorge für künftige Generationen auf die Zukunft gerichtet. Seine Effektivität wird von der Fortentwicklung im "Rio-Prozeß" abhängen.

Nachhaltige Entwicklung im internationalen Naturschutzrecht geht weit über die nachhaltige Nutzung von Ressourcen hinaus. Sie bestimmt sich nach dem Inhalt aller Regelungen des Übereinkommens über die biologische Vielfalt im Zusammenwirken mit den anderen geltenden naturschutzrechtlichen Übereinkommen auf universeller und regionaler Ebene. Daneben ist das in der Rio-Deklaration von 1992 enthaltene Konzept nur ein politisches Leitbild für eine zukünftige Entwicklungs- und Umweltpolitik.

Die Elemente des sustainable development haben Lücken im völkerrechtlichen Naturschutzinstrumentarium geschlossen und insbesondere die Nutzung von Ressourcen dem Grundsatz der Nachhaltigkeit unterworfen. Da andere Übereinkommen durch das Übereinkommen über biologische Vielfalt nicht verdrängt werden, bleibt der Schutz durch diese Verträge jedenfalls bestehen. Soweit nach dem Übereinkommen über die biologische Vielfalt ein erweiterter Schutz - etwa zugunsten des Schutzes der Vielfalt - umgesetzt wird, weil die biologische Vielfalt gefährdet ist, kommt eine Relativierung durch den Grundsatz der nachhaltigen Nutzung nicht in Betracht. Insofern wirkt sich nachhaltige Entwicklung im geltenden Naturschutzrecht nur insoweit relativierend aus, als es sich um für die Nutzung geeignete Ressourcen handelt, die nicht in ihrem Bestand bedroht sind.

\section{Regionales völkerrechtliches Naturschutzrecht}

Nach der Erörterung des universellen auch für Übersee geltenden internationalen Naturschutzrechts, seiner Entwicklung und der inhaltlichen Schwerpunkte, sind die in Übersee geltenden regionalen vertraglichen Naturschutzinstrumente darzustellen. Aus ihnen kann sich eine Fortentwicklung des bereits umrissenen Schutzes der biologischen Vielfalt oder des sustainable development ergeben. In weiterem Sinne gehört auch das regionale Völkervertragsrecht zum "Rio-Prozeß". 
Kein überseeisches Völkervertragsrecht, aber ein Vertrag, an den auch einige wenige afrikanische Staaten gebunden sind, ist das Übereinkommen über die Erhaltung der europäischen wildlebenden Pflanzen und Tiere und ihrer natürlichen Lebensräume (Berner Konvention) vom 19. September $1979 .^{89}$ Es enthält eine ausführliche Liste zu schützender, auch wandernder Tierarten. Damit ist es ein traditionelles Artenschutzinstrument, das insofern einen Aspekt schützt, der zwar auch der nachhaltigen Entwicklung zuzuordnen ist, aber in diesem Konzept zusammen mit anderen Schutzmaßnahmen aufgeht.

\section{a) Afrika}

In Afrika wurden früher als in anderen Regionen Naturschutzübereinkommen geschlossen. Schon im Jahre 1900 einigten sich die damaligen Kolonialmächte Großbritannien, Italien, Portugal, Spanien und Frankreich auf ein Übereinkommen zum Schutz wilder Tiere, Vögel und Fische in Afrika ${ }^{90}$ "zur Verhinderung unkontrollierter Tötung und zum Schutz verschiedener Arten nützlicher oder nicht aggressiver wilder Tiere in ihren afrikanischen Besitzungen." Das ursprüngliche Übereinkommen und das Nachfolgeübereinkommen von 1933 enthielten bereits eine Reihe moderner Instrumente zum Naturschutz. ${ }^{91}$

Die in Afrika geltenden regionalen völkerrechtlichen Übereinkommen zum Naturschutz sind sehr unterschiedlicher Natur. Einige Abkommen zielen nur in zweiter Linie auf Naturschutz, in erster Linie auf Kontrolle vor Plagen durch Heuschrecken ${ }^{92}$ oder sonstige Pflanzenschädlinge. ${ }^{93}$ Sie dienen der präventiven Kontrolle durch Schaffung von Koordinierungsbüros und mitgliedstaatliche Kontrolle. Nur wenige Bestimmungen zum Naturschutz

89

90

London Convention for the Protection of Wild Animals of 19 May 1900 (London); Text in: Bernd Rüster, Bruno Simma, Michael Bock (Hrsg.), International Protection of the Environment, 1975 ff., Bd. IV, S. 1607.

91

92

93 Sanitary Convention for Africa South of the Sahara; Kiss (ed.), Selected Multilateral Treaties (Fn. 35), S. 11, 115. 
enthält das Schiffahrts- und Zusammenarbeitsabkommen für das Niger-Tal vom 26. Oktober $1963^{94}$.

Demgegenüber handelt es sich bei dem seit 1969 in Kraft befindlichen, heute für 30 Staaten geltenden Afrikanischen Übereinkommen über die Erhaltung der Natur und der natürlichen Ressourcen vom 15. September $1968^{95}$ (African Nature Convention) um ein umfassendes Umweltabkommen, das neben genauen Definitionen Bestimmungen über den Schutz von Boden, Wasser, Fauna und Flora, geschützte Arten, Schutzgebiete, Umweltplanung, Umwelterziehung, Einrichtung von Umweltverwaltungen und zwischenstaatliche Zusammenarbeit enthält. Es sieht eine Unterscheidung zwischen zu schützenden und nutzbaren Ressourcen sowie eine Liste geschützter Arten vor. Es verpflichtet die Staaten zu Maßnahmen im Einklang mit Gewohnheitsrecht zum Schutz, der Nutzung und der Entwicklung der genannten Umweltmedien nach wissenschaftlichen Grundsätzen mit Rücksicht auf die Interessen der Bevölkerung.

Auf den Schutz der genetischen Vielfalt, den Schutz des Naturhaushalts und die nachhaltige Nutzung geernteter Naturschätze zielt das Protokoll über Schutzgebiete und wildwachsende Fauna und Flora in Ostafrika von $1985,{ }^{96}$ das im Rahmen der Nairobi-Konvention von $1985^{97}$ abgefaßt wurde. Übereinkommen und Protokoll sind bisher noch nicht in Kraft. $^{98}$ Das Protokoll nennt die Verpflichtung "to ensure the sustainable utilisation of harvested natural resources under their jurisdiction" (Art. 2 Abs. 1). Anders als die African Nature Convention verpflichtet das Protokoll zum Schutz gefährdeter Ökosysteme, zur nationalen Naturschutzplanung und der Berücksichtigung der traditioneller Betätigungen der lokalen Bevölkerung (Art. 2 und 12).

In vier Anhängen werden Maßnahmen zum Schutz wildlebender Pflanzenarten (Annex I), zum strengsten Schutz bedrohter wildlebender Pflanzenarten (Annex II und III) und zum Schutz wandernder Arten (Annex IV) vorgesehen. Anders als die oben genannten Übereinkommen ist das Hauptaugenmerk auf den Schutz der Natur, nicht ihre Nutzung oder Kontrolle gerichtet. Zudem ist es das erste afrikanische Übereinkommen über die biologische

94

Act Regarding Navigation and Economic Co-Operation Between the States of the Niger Basin, Niamey (1993); Kiss (ed.), Selected Multilateral Treaties (Fn. 35), S. 24, 186.

95 African Convention on the Conservation of Nature and Natural Resources, Algiers (1968); Kiss (ed.), Selected Multilateral Treaties (Fn. 35), S. 27, 207. Es stellt im weitesten Sinn eine Fortentwicklung des Übereinkommens von 1900 dar (vgl. Fn. 90),

Protocol Concerning Protected Areas and Wild Fauna and Flora in the Eastem African Region vom 21. Juni 1985, International Environmental Legal Materials and Treaties (I.E.L.M.T.) 985:47.

Nairobi Convention for the Protection, Management and Development of the Marine and Coastal 98 Environment of the East African Region vom 21. Juni 1985, I.E.L.M.T. 985:46.

Vgl. dazu Sands, Principles (Fn. 12), S. 300, 389. 
Vielfalt, das zudem mit einer Vertragstaatenkonferenz ein Kontrollgremium zur Begutachtung der Erfüllung der Verpflichtungen aus dem Vertrag geschaffen hat. ${ }^{99}$

Eine Besonderheit in Afrika ist die erste völkerrechtliche Kodifikation eines Menschenrechts auf Natur- bzw. Umweltschutz in Art. 24 der Banjul-Charta: ${ }^{100}$ "All people shall have the right to a general satisfactory environment favorable to their development." Es entspricht der schon in der Stockholmer Deklaration von 1972 (Prinzip 1) ausgedrückten menschenrechtlichen Dimension des Umweltschutzes. In der Banjul-Charta ist auch das Recht auf Entwicklung enthalten (Art. 22), das seit Stockholm und erneut in Rio im Zentrum der Diskussion des Konzepts eines sustainable development stand. ${ }^{101}$

b) Asien

Für den regionalen völkervertraglichen Naturschutz ist in Asien neben drei internationalen Übereinkommen zur Kontrolle von Naturplagen ${ }^{102}$ und einem Pflanzenschutzabkommen ${ }^{103}$ insbesondere auf das Übereinkommen über Naturschutz im Südpazifik vom 12. Juni $1976^{104}$ (Apia-Konvention) und das in Kuala Lumpur unterzeichnete ASEAN-Übereinkommen über den Schutz von Natur und Naturgütern vom 9. Juli $1985^{105}$ einzugehen.

Die Apia-Konvention ist für 5 Staaten in Kraft und verpflichtet zur Ausweisung von Schutzgebieten, zu wissenschaftlicher Zusammenarbeit und zu Zusammenarbeit bei Aus-

99

100

Vgl. dazu Sands, Principles (Fn. 12), S. 390.

African Charter on Human and Peoples' Rights, Text in: Henry J. Steiner / Philip Alston, International Human Rights in Context, Oxford 1996, S. 1215.

101 Vgl. zum Recht auf nachhaltige Entwicklung Odendahl, Das Recht auf Entwicklung (Fn. 13), S. 266.

102 Abkommen zur Gründung einer Kommission zur Bekämpfung der Wüstenheuschrecken in Südwest-Asien vom 3. Dezember 1963; Agreement for the Establishment of a Commission for Controlling the Desert Locust in the Eastern Region of its Distribution Area in South-West Asia, Rome (1963); Kiss (ed.), Selected Multilateral Treaties (Fn. 35), S. 25, 190. - Abkommen zur Gründung einer Kommission zur Bekämpfung der Wüstenheuschrecken im Nahen Osten vom 2. Juli 1965; Agreement for the Establishment of a Commission for Controlling the Desert Locust in the Near East, Rome (1965); Kiss (ed.), Selected Multilateral Treaties (Fn. 35), S. 26, 198.

103 Pflanzenschutzabkommen für den südostasiatischen und pazifischen Raum vom 27. Februar 1956; Plant Protection Agreement for the South East Asia and Pacific Region, Rome (1956); Kiss (ed.), Selected Multilateral Treaties (Fn. 35), S. 12, 117.

104 Convention on Conservation of Nature in the South Pacific, Apia (1976); Kiss (ed.), Selected Multilateral Treaties (Fn. 35), S. 47, 463.

105

ASEAN Agreement on the Conservation of Nature and Natural Resources, Kuala Lumpur (1985); Sands et al. (eds.), Documents (Fn. 24), S. 958. 
bildung und Öffentlichkeitsarbeit. Nach der Ausweisung dürfen die Grenzen eines Nationalparks nur unter sehr engen Voraussetzungen verändert werden.

Noch nicht in Kraft getreten ist das ASEAN-Übereinkommen. ${ }^{106}$ Es ist ein sehr weit fortentwickeltes Naturschutzübereinkommen, das klarer als das Übereinkommen über die biologische Vielfalt den Unterschied zwischen dem - vorrangigen - Schutz der Artenvielfalt durch Schutzgebiete (Art. 13), Verbote und Genbanken (Art. 3) einerseits und der nachhaltigen Nutzung von Arten im Rahmen eines Nutzungsmanagements (Art. 4) andererseits unterscheidet, schließlich als dritte Schutzkategorie den Artenschutz einschließlich des Handels mit Arten behandelt (Art. 5).

Ein eigener Artikel ist der Bedeutung der Pflanzendecke und den Waldgebieten gewidmet (Art. 6). Des weiteren sind Vorschriften zum Bodenschutz und zum Schutz von Wasser und Luft (Art. 7 -9) ebenso enthalten wie zur Kontrolle von Emissionen und der Förderung umweltverträglicher industrieller Verfahren und Produkte (Art. 11). Die Pflicht zur Durchführung einer Umweltverträglichkeitsprüfung (Art. 12), zu Umwelterziehung (Art. 16) und der Aufbau einer Umweltverwaltung (Art. 17) sind vorgesehen. In Art. 9 wird im Zusammenhang mit der Luftreinhaltung ausdrücklich auf die Vereinbarkeit mit dem sustainable development Bezug genommen.

Das ASEAN-Übereinkommen von Kuala Lumpur wurde 7 Jahre vor der Konferenz von Rio de Janeiro formuliert und setzt die bis dahin gefestigten Überlegungen zum sustainable development überzeugend um. Nur wegen der zeitlichen Vorlagerung verbietet es sich, das Übereinkommen als Fortführung des Geistes von Rio zu apostrophieren.

\section{c) Lateinamerika}

In Lateinamerika bestehen im wesentlichen drei Instrumente zum Schutz der Natur: Das Internationale Übereinkommen zum Schutz von Flora, Fauna und naturnaher Landschaftsbilder in der westlichen Hemisphäre vom 12. Oktober 1940, ${ }^{107}$ dem derzeit von 21 lateinamerikanischen Staaten und den USA 19 Staaten beigetreten sind, der Vertrag über Zusammenarbeit im Amazonas-Gebiet vom 3. Juli $1978^{108}$ sowie der Vertrag zwischen

106 Bisher haben nur Indonesien, Philippinen und Thailand Vertragsparteien, Brunei Darrusalam, Malaysia und Singapur haben unterzeichnet.

107 Convention on Nature Protection and Wildlife Preservation in the Western Hemisphere, Washington (1940); Sands et al. (eds.), Documents (Fn. 24), S. 899.

108 Treaty for Amazonian Cooperation, Brasilia (1978); Sands et al. (eds.), Documents (Fn. 24), S. 936. 
Bolivien und Argentinien über Naturschutz und die Integration von Naturparks in Grenznähe vom 16. März 1996. ${ }^{109}$

Das Übereinkommen über den Schutz der westlichen Hemisphäre verpflichtet die Vertragsparteien zur Errichtung von Nationalparks und sonstigen Schutzgebieten, die teilweise als Totalreservate auszugestalten sind (Art. II Abs. 1). Verboten sind in diesen Gebieten wirtschaftliche Nutzungen, Handel und Jagd (Art. III). Vorgesehen sind in dem Übereinkommen auch Vorschriften zum Schutz aller Pflanzen und Tiere außerhalb von Schutzgebieten (Art. V bis VII) mittels Listen zu schützender Arten. Eine Schwierigkeit der Konvention ist das hohe Vollzugsdefizit und das Fehlen von Kontrollmechanismen und -instanzen. ${ }^{110}$

Nur geringe Bedeutung für den internationalen Naturschutz besitzen die nur sehr allgemein formulierten Bestimmungen des Amazonas-Vertrages von 1978. ${ }^{111}$ Der Schwerpunkt des Vertrages liegt auf wirtschaftlicher Nutzung. Die Umweltschutzbestimmungen beschränken sich auf allgemeine Zielbestimmungen sowie Informations- und Wissenschaftsaustausch.

Das fortschrittlichste Vertragswerk in Lateinamerika ist das Protokoll über Schutzgebiete und wildlebende Arten ${ }^{112}$ zum Übereinkommen von 1983 von Cartagena de Indias (Kingston Protokoll). Es ist von 13 karibischen, latein- und nordamerikanischen Staaten, Frankreich, den Niederlanden und Großbritannien unterschrieben, aber noch nicht in Kraft. ${ }^{113}$ Mit dem Ziel der Erhaltung produktiver Ökosysteme verpflichten sich die Vertragsparteien gefährdete Gebiete, sowie Arten von Tieren und Pflanzen unter Schutz zu stellen und eine nachhaltige Nutzung zu gewährleisten (Art. 3 Abs. 1). Listen im Anhang der Konvention bestimmen die Prioritäten beim Schutz von Flora und Fauna. Damit enthält es nur einige wenige Elemente eines sustainable development. Insoweit kommt dem Übereinkommen über biologische Vielfalt eine gesteigerte Bedeutung zu, insbesondere bis zum Inkrafttreten des Protokolls.

109

Bernd Rüster, Bruno Simma, Michael Bock (Hrsg.), International Protection of the Environment, 1975 ff., Bd. XXV, S. 296.

110 Vgl. dazu Sands, Principles (Fn. 12), S. 391.

111 Treaty for Amazonian Co-operation of 3 July 1978 (Brasilia); Sands et al. (eds.), documents (Fn. 24), S. 936.

112 Kingston Protocol Concerning Specially Protected Areas and Wildlife of 18 January 1990, YbIEL 1 (1990), S. 441.

113 Vgl. dazu Sands, Principles (Fn. 12), S. 393. 
Die regionalen völkerrechtlichen Verträge zum Schutz der Natur in den drei behandelten überseeischen Regionen markieren - wenn auch weniger ausgeprägt - die Entwicklung, die sich am universellen Vertragsrecht nachzeichnen lies. Im Ergebnis ist eine Ausweitung des Schutzes in Hinblick auf die Schutzgüter, die Schutzinstrumente, begleitende Informationsund Kooperationspflichten, teils planerische Komponenten vorgesehen. Bisher ist kein Vertrag in Kraft, der wesentliche Elemente des Konzepts der nachhaltigen Entwicklung bereits enthält. Das Ostafrika-Protokoll, das ASEAN-Übereinkommen und das KingstonProtokoll enthalten bereits entscheidende Elemente für eine nachhaltige Entwicklung. Die Übereinkommen sind jedoch nicht in Kraft und die einzelnen Bestimmungen bleiben, mit Ausnahme der Vorschriften des ASEAN-Übereinkommens, deutlich hinter dem Übereinkommen über die biologische Vielfalt zurück.

\section{Völkergewohnheitsrecht}

Sieht man einmal vom nachbarrechtlichen Schädigungsverbot ab, das auch in Art. 3 des Übereinkommens über biologische Vielfalt enthalten ist, ist dem Völkergewohnheitsrecht kein für das Naturschutzrecht geltender Rechtssatz zu entnehmen. ${ }^{114}$

Die Gründe scheinen offenbar. Da das Umweltvertragsrecht in Bezug auf den Naturschutz jeweils verhältnismäßig schnell auf neue Entwicklungen reagieren muß ${ }^{115}$ und reagiert hat, war der zeitliche Rahmen für die Bildung einer gemeinsamen, von den Vertragsverhandlungen losgelösten Staatenpraxis - über eine gewisse Dauer hinweg mit entsprechender Rechtsüberzeugung - zu kurz. ${ }^{116}$ Das sich nur langsam fortentwickelnde Völkergewohnheitsrecht kann in solchen Fällen die rechtlichen Entwicklungen allenfalls nachvollziehen. Zudem besteht, etwa in Hinblick auf die Konzepte nachhaltiger Entwicklung und nachhaltiger Nutzung, zwischen den Staaten noch immer ein zu großer Dissens in Hinblick auf die Bestimmung des Inhalts. ${ }^{117}$ Es konnte daher - auf völkervertraglicher Ebene-lediglich die Formulierung des Konzeptes nachhaltiger Entwicklung im konkreten Einzelfall vorgenommen werden.

Auch das Konzept des sustainable development, so wie es in der Rio-Deklaration enthalten ist, ist noch nicht zu Völkergewohnheitsrecht erstarkt. Es mangelt an hinreichender Staa-

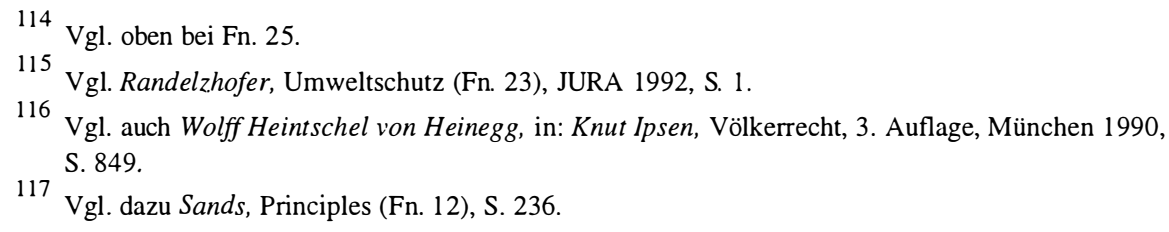


tenpraxis. Diese kann auch nicht durch die Beteiligung einer Vielzahl von Staaten an internationalen Konferenzen oder dortige inhaltliche Aussagen und Abstimmungen ersetzt werden. $^{118}$

Die Deklaration, die als Beschluß der Staatenkonferenz und nicht als völkerrechtlicher Vertrag unterzeichnet worden ist, gehört zu der großen Zahl rechtlich nicht verbindlicher Dokumente jenseits von Völkervertrags- und -gewohnheitsrecht, in denen zentrale Aussagen zum völkerrechtlichen Umweltrecht - auch zum völkerrechtlichen Naturschutzrecht enthalten sind. Hierzu zählen - neben der Rio-Deklaration für Umwelt und Entwicklung jedenfalls auch die Agenda 21 vom 16. Juni $1992^{119}$ sowie die Wald-Deklaration vom 13. Juni 1992. ${ }^{120}$ Ihnen kommt für die Auslegung von Völkervertragsrecht eine wichtige, wenn auch nur mittelbare, Bedeutung $\mathrm{zu}^{121}$ auch weisen sie die Richtung für das zukünftige völkerrechtliche Umweltrecht, führen möglicherweise zur Entwicklung von differenzierterem Völkergewohnheitsrecht auf dem Gebiet des Umweltrechts.

Für die Zukunft kommen einzelne Aspekte des völkerrechtlichen Naturschutzes für eine völkergewohnheitsrechtliche Konkretisierung und Verfestigung in Betracht. $\mathrm{Zu}$ nennen sind das Vorsorgeprinzip, die Ökozentrik des völkerrechtlichen Umweltschutzes oder das Entfallen der ,Erforderlichkeit“ erheblicher Schadenszufügung beim allgemeinen Schädigungsverbot. ${ }^{12}$

\section{Schluß}

Heute besteht ein modernes Recht der natürlichen Lebensbedingungen im Völkerrecht, das - mit einer Vielzahl regionaler Instrumente - auch auf die spezifischen ökologischen, ökonomischen und sozialen Besonderheiten der Staaten in Übersee reagiert hat und von dem auch in Zukunft zu erwarten ist, daß es sich zur Anpassung und Einbeziehung, und damit zur effektiven und verbindlichen Umsetzung neuer Umweltschutzziele eignet. Bei der Schaffung neuer vertraglicher Instrumente kann auf das kontinuierlich fortentwickelte Instrumentarium zurückgegriffen werden. - Es hat sich gezeigt, daß selbst ein Paradig-

118 Ebenso Beyerlin / Marauhn, Rechtsetzung (Fn. 15), S. 8 f.

119 Agenda 21, Rio de Janeiro (1992), UN Doc. A/CONF. 151/26; Sands et al. (eds.), Documents (Fn. 24), S. 55.

120

Non-legally Binding Authoritative Statement of Principle for a Global Consensus on the Management, Conservation and Sustainable Development of all Types of Forests, Rio de Janeiro (1992), UN Doc. A/CONF. 151/6/Rev. 1; Sands et al. (eds.), Documents (Fn. 24), S. 874.

121 Vgl. Beyerlin / Marauhn, Rechtsetzung (Fn. 15), S. 8, sowie Randelzhofer, Umweltschutz (Fn. 23), JURA 1992, S. 2.

122 Vgl. auch Heintschel von Heinegg, in: Ipsen, Völkerrecht (Fn. 116), S. 849. 
menwechsel durch die Einfügung neuer Elemente in das bestehende Naturschutzrecht bewältigt werden kann.

Das Konzept der nachhaltigen Entwicklung ist so alt wie meine Eingangsbemerkung: In der World Conservation Strategy von 1980, der auch mein Eingangszitat entnommen war, war erstmals von "sustainable development" die Rede. Es heißt dort, eine bestandsfähige Entwicklung könne nur durch Erhaltung der wesentlichen ökologischen Prozesse und der lifesupport-Systeme erreicht werden, durch Schutz der genetischen Vielfalt und durch Sicherstellung einer nachhaltigen Nutzung ("sustainable utilization") von Arten und Ökosystemen. Zwei zunächst einander fremde rechtliche Stränge könnten in diesem Konzept konvergieren, sofern es gelingt, bei Fragen der Entwicklung - gleich einem Prinzip ökologischer Verhältnismäßigkeit - die staatliche Souveränität durch die Pflicht zu hinreichendem Schutz und nachhaltiger Nutzung zu beschränken. Die angesprochenen jüngeren völkerrechtlichen Verträge versuchen dies - global und in Übersee.

Auch wenn durch das Konzept des sustainable development die Nachwelt ${ }^{123}$ nicht mit eigenen Rechten ausgestattet wird, bleibt zu hoffen, daß die Kodifizierung des Konzepts der nachhaltigen Entwicklung im Naturschutzrecht für die Nachwelt zumindest ein Minimum an bewahrter oder gepflegter Natur erhält.

Die dem universellen Völkerrecht vorangegangene regionale naturschutzrechtliche Umsetzung des Konzepts der nachhaltigen Entwicklung im Ostafrika-Protokoll von 1985 und in der ASEAN-Konvention von 1985, teilweise im Kingston-Protokoll von 1990, nimmt der Kritik an der Haltung der Staaten in Übersee zum völkerrechtlichen Naturschutz die Spitze und läßt - zu Gunsten der nachhaltigen Entwicklung - für den "Rio-Folgeprozeß" diesseits und Über-See hoffen .

${ }^{123}$ Zum Nachweltschutz vgl. Anthony' d'Amato, Do We Owe a Duty to Future Generations to Preserve the Global Environment?; Edith Brown Weiss, Our Rights and Obligations to Future Generations for the Environment; Lothar Gündling, Our Responsibility to Future Generations, alle in AJIL 84 (1990), 190, 198 bzw. 207. 


\title{
The Relationship between Law and Religion in Islamic Law
}

\author{
By J. Christian Wichard
}

Shari'a, Islamic law, is closely linked to law and to religion. However, the first question to be answered is, what is Islamic law? After a first introduction to specific traits of Shari'a, five examples show different legal aspects of Islamic law as a whole.

The first aspect discussed is that Islamic law is revealed law which leads to an inseparable link between law and religion - with few exceptions. As a second aspect, it has to be noted that the deduction of law by lawyers does not lead to an absolute result, or absolute truth, but is only of relative relevance to the specific decision. The relativity of law and the limits to human fact and law finding leads to a demand for objective criteria to support a legal decision and to avoid arbitrary verdicts, one of the reasons of the greater importance attributed to external facts as compared to motives and intentions. Fourth, the fact that man can only decide on objective facts, but not internal motives, leads to a differentiation between norms sanctioned here and others sanctioned "hereafter". The fifth aspect pointed out is the equality between Christians and Muslims in some areas of the law of contracts. The author concludes, inter alia, that although the given objective order is not touched there is room for new solutions along the fringes - outside the normative order. On the one hand, this shows how the development of law is slowed down by the religious link, on the other hand, Shari'a is misunderstood to be much more strict, if separated from its religious background, which is the basis for its understanding.

\section{Conservation of Nature by International Law and Sustainable Development in Overseas Countries}

\section{By Ulf Marzik}

The notion of sustainable development, which has been introduced by the World Conservation Strategy of 1980, may be considered one of the key expressions in international relations and, especially, in international environmental law. It addresses one of the most pressing issues the international community is facing today: the conflict between the protection of the environment and the use of natural resources for further development.

The 1992 Convention on Biological Diversity is the first global convention on the conservation of nature to include the concept of sustainable development in its provisions. Primarily, the convention is focussing on a specific aspect of nature conservation, the protection of biological diversity, and is thus promoting the conservation of nature by international law to meet this newly acknowledged threat to the ecosystem. The article, therefore, outlines the development of nature conservation by international treaties from 
Stockholm to Rio, both on a global level and regarding Africa, Asia and Latin America. In a larger sense, the Biodiversity Convention is also designed to put an end to the piecemeal approach which characterized nature conservation by international law in the past decades, not by substituting the other conventions, but by adding missing links and a common background. The concept of sustainable development as embodied in the Convention on Biological Diversity is such a link addressing the conflict between environment and development: It includes the concept of protection of species and their "variety" for future generations (intergenerational equity) within and outside of ecosystems and natural habitats, the duty to sustainable use natural resources, the concept of transfer of technology and equitable sharing of scientific knowledge and financial burdens, and the integration of biodiversity conservation schemes into national plans, programs and projects. Thus the concept of sustainable development, in the context of international environmental law on nature conservation, is far more than the concept of sustainable use which is part of it.

\section{Constitutional Law and Traditional Social Order in Southern Pacific Micro-States}

\section{By Jörg Menzel}

The South Pacific Region boasts more constitutional "per capita" than any other region in the world today. Fourteen states and a number of dependent territories are inhabited by only a few million people. The diversity of current constitutional structures reflect the history of the region. In general, "pre-contact-societies" in Melanesia were small and quite egalitarian whereas those of Micronesia and especially those of Polynesia tended to be highly stratified proto-states. With the arrival of Europeans and (subsequent) colonialisation, European legal systems were implemented throughout the region. This does not mean, however, that the legal traditions of the indigenous peoples have disappeared. Particularly in "indirect-rule"-systems as Fiji and Western Samoa legal traditions were to a certain extent accepted. Colonial authorities even confirmed the status of indigenous authorities on some occasions. These authorities (usually called "chiefs") also play an important role within in the current political systems. This is not only the fact in the monarchy of Tonga or in aristocratic Western Samoa, but also in Fiji, Vanuatu and elsewhere. Since decolonialisation attempts have also been made to strengthen the role of indigenous customary law within the legal system of the region. Customary law is recognized as a source of law within many constitutions, it is absolutely predominant within the area of land tenure. The tension between traditional rule and village life on the one hand, and the modern idea of democracy and individual human rights on the other make "the politics of tradition" particularly controversial, not only in highly stratified societies like Western Samoa, Tonga, and Fiji, but in the region as a whole. 